\title{
Palaeolithic archaeology and 3D visualization technology: recent developments
}

\author{
Julien Riel-Salvatore, Myungsoo Bae, Peter McCartney \& Anshuman Razdan*
}

This paper presents computer-aided lithic analysis conducted in the context of an interdisciplinary research framework combining computer science and archaeology - the Partnership for Research in Stereo Modeling (PRISM), at Arizona State University (<http://prism.asu.edu>, <http://3dk.asu.edu $>$ ). Pilot projects conducted as part of PRISM serve to find real-world applications to the tridimensional visualization technology developed as part of the project. Here, we focus on one of these projects which aims at automating the lithic refitting process.

The artefacts used in this study are refitted Ahmarian cores collected at Wadi Hasa Locality 623X (Lindly et al. 2000) and curated at Arizona State University. Using previously refitted material allows us to know exactly which pieces refit to which, thereby giving us an external way of testing the results of the refitting software. As well, it provides an estimate of the time involved in doing the refit manually and, by extension, a baseline for how rapid the automatic program would have to be in order usefully to substitute manual efforts.

3D models of those artefacts were created by scanning each piece using a Cyberware M15 or M3030 Laser Digitizer (FIGURE 1). This generates a cloud of points, each of which is defined by $x$, $\mathrm{y}, \mathrm{z}$ coordinates. The points are then topologically connected together through the process of triangulation, which results in a 'mesh-frame' from which a smooth surface is extrapolated; this system of surface generation is common in computer imaging. The scanned lithics are then imported into Raindrop Geomagic 4.0 to remove noise and unwarranted scanning artefacts. The data are also reduced using polygon decimation to make the models wieldier to analyse.

Once adequately formatted, a model can be imported into analytical programs developed at the PRISM laboratory (FIGURE 2). The first, feature segmentation software, delineates regions by identifying high and low geometrical points on the model, given a user-specified sensitivity level which can be adjusted to obtain finer or coarser region definition.
The second program is Region Editor; it permits the merging and splitting of regions originally highlighted by the surface segmentation software to transform them into analytically meaningful surfaces (e.g., a flake's ventral surface). It also allows the user to save these new regions as analytical surfaces which can subsequently be used in the automated refitting process. On top of normal linear measurements (i.e., length, width, thickness), Region Editor also allows the derivation of information such as total object volume, absolute object symmetry, a surface's area, and the average angle at which surfaces intersect. All these represent attributes that researchers traditionally have had difficulty to measure accurately.

As concerns the automatic refitting itself, we have attempted several methods (FIGURE 3). One proved successful, albeit slow and very userdependent; in effect, this severely limits the usefulness of that approach since our goal is to reduce the time and effort involved in refitting. We are currently developing alternative shapematching software that extracts several sets of point configuration per surface so as to detect complementary surfaces on lithic material. Though not yet conclusive, this method appears quite promising.

While an automated lithic refitting software package is not yet fully operational, tangential applications of the software developed as part of PRISM already warrant presentation and consideration for current approaches to lithic analysis. We have already described some of the varied and accurate measurements $3 \mathrm{D}$ visualization software permits. Additionally, such software allows the greater dissemination of archaeological data while creating durable and flexible records of artefacts, including those currently protected by national antiquities acts or under threat of repatriation (RielSalvatore et al. 2002).

Acknowledgements. Many thanks to Prof. G.A. Clark for giving us access to the $623 \mathrm{X}$ material. Support for this research provided by NSF grants IIS-998016 (3D Knowledge) and BNS-8406601 (to GAC), and SSHRCC grant 752-20010460 (to JR-S).

* Partnership for Research in Stereo Modeling, Arizona State University, PO Box 875906, USA. julienrs@asu.edu 


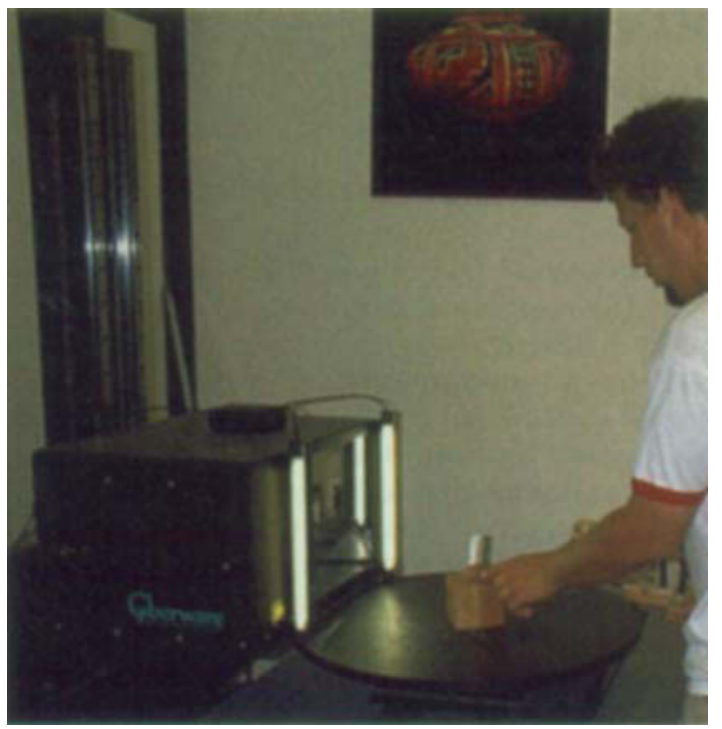

\section{References}

LINDLY, J.M., R.F. BECK \& G.A. CLARK. 2000. Core reconstructions and lithic reduction sequences at WHS $623 \mathrm{X}$ : an

Upper Palaeolithic site in the Wadi al-Hasa, West-Central Jordan, in N.R. Coinman (ed.), The archaeology of the Wadi al-Hasa, West-central Jordan 2: 211-26. Tempe (AZ): Arizona State University. ASU Anthropological Research Papers.

Riel-Salvatore, J., M. Bae, G.A. Clark, J. Lindly, P. MCCARTNEY \& A. RAZDAN. 2002. The past meets the future: $3 \mathrm{D}$ visualization technology and lithic analysis at Wadi al-Hasa Locality 623X, Jordan, Journal of Human Evolution 42(3): A39.

FIGURE 1 (left). 3D scanner with artefact; a researcher indicates where the laser hits the object.

FIGURE 2 (below). Analysis: flake processed with feature segmentation software (at left), and Region Editor (at right).

FIGURE 3 (bottom). Results: 3D models of a flake and core, with matching surfaces.
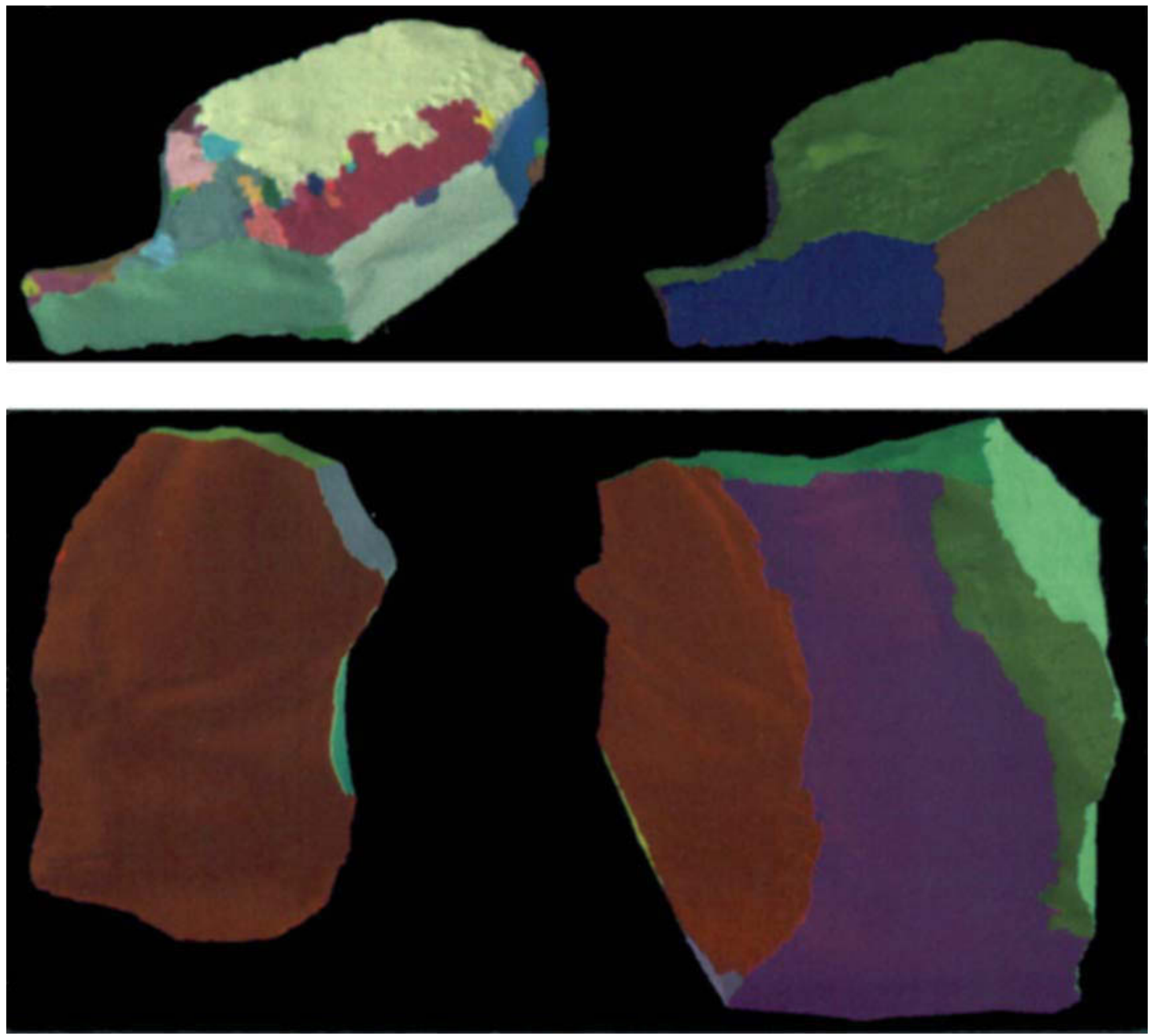